\title{
Article \\ Effects of Various Mineral Trioxide Aggregates on Viability and Mineralization Potential of 3-Dimensional Cultured Dental Pulp Stem Cells
}

\author{
Seung-Ho Kwon ${ }^{1}$, Hyun-Jeong Jeong ${ }^{2}$, Bin-Na Lee ${ }^{3}$, Hyo-Seol Lee ${ }^{4}$, Hyun-Jung Kim ${ }^{1}{ }^{\oplus}$, Sun-Young Kim ${ }^{5}$, \\ Duck-Su Kim ${ }^{2}$ and Ji-Hyun Jang ${ }^{2, *}$ \\ 1 Department of Conservative Dentistry, Kyung Hee University Dental Hospital, Seoul 02447, Korea; \\ pokd92@gmail.com (S.-H.K.); kimhyunjung@khu.ac.kr (H.-J.K.) \\ 2 Department of Conservative Dentistry, School of Dentistry, Kyung Hee University, Seoul 02447, Korea; \\ jhj7662@naver.com (H.-J.J.); dentist96@khu.ac.kr (D.-S.K.) \\ 3 Department of Conservative Dentistry, School of Dentistry, Dental Science Research Institute, Chonnam \\ National University, Gwang-ju 61186, Korea; bnlee13@jnu.ac.kr \\ 4 Department of Pediatric Dentistry, School of Dentistry, Kyung Hee University, Seoul 02447, Korea; \\ snowlee@khu.ac.kr \\ 5 Department of Conservative Dentistry and Dental Research Institute, School of Dentistry, Seoul National \\ University, Seoul 03080, Korea; denkim@snu.ac.kr \\ * Correspondence: jangjihyun@khu.ac.kr; Tel.: +82-2-958-9330
}

check for

updates

Citation: Kwon, S.-H.; Jeong, H.-J.; Lee, B.-N.; Lee, H.-S.; Kim, H.-J.; Kim, S.-Y.; Kim, D.-S.; Jang, J.-H. Effects of Various Mineral Trioxide Aggregates on Viability and Mineralization Potential of 3-Dimensional Cultured Dental Pulp Stem Cells. Appl. Sci. 2021, 11, 11381. https://doi.org/ 10.3390/app112311381

Academic Editor: Vittorio Checchi

Received: 2 November 2021

Accepted: 29 November 2021

Published: 1 December 2021

Publisher's Note: MDPI stays neutral with regard to jurisdictional claims in published maps and institutional affiliations.

Copyright: (c) 2021 by the authors. Licensee MDPI, Basel, Switzerland. This article is an open access article distributed under the terms and conditions of the Creative Commons Attribution (CC BY) license (https:// creativecommons.org/licenses/by/ $4.0 /)$.

\begin{abstract}
Three-dimensionally (3D) cultured dental pulp stem cells (DPSCs) reportedly exhibit superior multi-lineage differentiation capacities and have a higher expression in regeneration-related gene categories compared to conventionally cultured DPSCs. This study aimed to evaluate the effects of various mineral trioxide aggregates (MTAs) on DPSCs cultured in 3D, assessing their cell viability and tissue mineralization properties. We examined the morphology, cell viability, alkaline phosphate (ALP) activity and qualitative alizarin red S staining assay of the DPSCs that reacted with various MTAs, which included ProRoot (PRM), Biodentine (BIO), and Well-Root PT (WRP), in two different culture plates, an ultra-low attachment plate (ULA) and a conventional monolayer plate (2D). As a control, MTA-free and IRM samples were prepared. None of the MTA groups affected the microsphere-forming characteristics of DPSCs that had been cultured in ULA. The DPSCs that were cultured in ULA showed high cell viability in all MTA groups compared to IRM. The mineralization potential was favorable in all MTA groups, with a significantly higher ALP activity among the DPSCs that were cultured in ULA. Among MTAs, the PRM group showed substantially higher ALP activity than the other MTA groups. In conclusion, our results indicate that 3D-cultured DPSCs with various MTAs showed comparable viability and mineralization capacity similar to those cultured without reacting with MTA cement.
\end{abstract}

Keywords: mineral trioxide aggregates; dental pulp; restorative dentistry; culture method

\section{Introduction}

Mineral trioxide aggregates (MTAs) are biocompatible endodontic cements that are mainly composed of calcium and silicate elements [1]. MTAs were first developed and introduced as a root-end filling and perforation repair material [2,3], and they might also be useful for pulp capping, pulpotomy, apexification, apexogenesis, and root canal filling [4]. MTAs are a biocompatible material that are less cytotoxic than conventional endodontic materials, and they have the advantages of promoting the formation of mineralized tissue and preventing microleakage $[5,6]$.

ProRoot MTA (PRM; Dentsply Sirona, Tulsa, OK, USA) is the first commercially available MTA for clinical use and is applied by mixing calcium silicate-based powder with distilled water [7]. However, PRM has several drawbacks, including a prolonged setting 
time, difficulty in handling, and discoloration. To overcome these disadvantages, various types of improved MTA products have been developed. Biodentine (BIO; Septodont, Saint Maur des Faussés, France) has a short setting time and improved handling properties because of the incorporation of a hydrosoluble polymer and calcium chloride in the liquid [8]. Well-Root PT (WRP; Vericom, Chuncheon, Korea) is a recently introduced MTA that is a ready-to-use, premixed, bioceramic paste that contains polyethylene glycol and polypropylene glycol. These different options for MTAs were evaluated in the present study to understand their strengths and limitations as options for clinical endodontic procedures.

Regenerative endodontics is defined as a biologically based procedure that is designed to replace damaged structures, such as dentin, root structures, and cells of the pulp-dentin complex [9]. The development of regenerative endodontics requires research on the correct spatial assembly of stem cells, growth factors, and tissue engineering scaffolds [10]. Various dental stem cells, including stem cells from exfoliated deciduous teeth (SHEDs), stem cells from apical papilla (SCAPs), and periodontal ligament stem cells (PDLSCs), which are situated around the dental tissues, have been used for regenerative endodontics [11]. Dental pulp stem cells (DPSCs) are thought to be a superior source of multipotent mesenchymal stem cells (MSCs) [12]. DPSCs have attracted considerable interest because of their potential for use in regenerative endodontics and their easy availability in vital pulp tissues [13].

From the perspective of tissue engineering, data on stem cells have mostly been gathered through the study of cells that have been cultured as a two-dimensional (2D) monolayer on a dish surface [14]. DPSCs have also been cultured on 2D monolayers in previous in vitro studies [15]. However, 2D cultures may not reflect the in vivo microenvironment of stem cells, and this may lead to the loss of some of their stemness properties [16]. In regenerative endodontics, DPSCs come into contact with biocompatible materials, such as MTAs, in a three-dimensional (3D) pulp space. Recently, various 3D culture methods, including the use of microspheres, have been developed to overcome the limitations of monolayer cultures. DPSCs that have been cultured in microsphere-forming plates have presented superior multilineage differentiation capacities and have demonstrated a higher expression of regeneration-related genes compared to those cultured in a conventional monolayer plate [12,16].

The aim of this study was to evaluate the effects of various MTAs on DPSCs that have been cultured in $3 \mathrm{D}$, assessing their cell viability and tissue mineralization properties. We examined the morphology, viability, and alkaline phosphate (ALP) activity of DPSCs that reacted with various MTAs in vitro.

\section{Materials and Methods}

\subsection{Cell Culture}

The primary cultures of DPSCs that were isolated from extracted human teeth at passage three (Cefobio, Seoul, Korea) were cultured in a basal medium that contained alpha minimum essential medium ( $\alpha$-MEM; GIBCO, Carlsbad, CA, USA), $10 \%$ fetal bovine serum (FBS; Corning, New York, NY, USA), and $100 \mathrm{U} / \mathrm{mL}$ penicillin/streptomycin (GIBCO) in a $95 \%$ humidified atmosphere with $5 \% \mathrm{CO}_{2}$ at $37^{\circ} \mathrm{C}$, and the medium was changed every two days. A subculture of the cells was created when they reached confluence.

For the 2D monolayer culture (2D group), a subculture of DPSCs was developed in a $100 \mathrm{~mm}$ culture dish (2D plate; Corning, NY, USA). For this, DPSCs were treated with $0.25 \%$ trypsin-EDTA (GIBCO, NY, USA) for $3 \mathrm{~min}$, collected, centrifuged at $1500 \mathrm{rpm}$ for $3 \mathrm{~min}$, and seeded in a 24-well cell culture plate (SPL Life Science, Seongnam, Korea) at a density of $1.0 \times 10^{4}$ cells/well. For the 3D microsphere culture (ULA group), a subculture of DPSCs was developed in Corning ${ }^{\circledR}$ Ultra-Low Attachment 96-well plate (Corning) following the same protocol as for the $2 \mathrm{D}$ group, but seeded at a density of $2.0 \times 10^{4}$ cells/well.

\subsection{Preparation of MTA Specimens and MTA Cement Eluates}

The MTAs that were used in this study were PRM, BIO, and WRP; their compositions are listed in Table 1. The cements were prepared using sterile instruments following the 
manufacturers' recommendations. Different samples of PRM, BIO, and WRP were shaped into $2 \mathrm{~mm}$ thick disks with a diameter of $7 \mathrm{~mm}$ using rubber molds. As a negative control, Intermediate Restorative Material (IRM; Dentsply Sirona) was prepared in the same manner. After preparation, the samples were placed in 2D plates and hydrated with humidified gauze at $37^{\circ} \mathrm{C}$ for 5 days to allow for complete setting. Before extracting the cement eluate, the materials were sterilized through exposure to ultraviolet light for $30 \mathrm{~min}$, and then each specimen was separately soaked for $5 \mathrm{~d}$ in $20 \mathrm{~mL}$ of $\alpha$-MEM without FBS at $37^{\circ} \mathrm{C}$ and $5 \% \mathrm{CO}_{2}$ under $95 \%$ humidity. After $5 \mathrm{~d}$, the media were collected, filtered through sterile $0.22 \mu \mathrm{m}$ filters, and diluted at a 1:1 ratio before testing on the cells was conducted.

Table 1. Materials used in this study.

\begin{tabular}{|c|c|c|c|}
\hline Group & Product Name & Manufacturer & Chemical Composition \\
\hline IRM & $\begin{array}{c}\text { Intermediate } \\
\text { Restorative Material }\end{array}$ & $\begin{array}{l}\text { Dentsply Sirona, } \\
\text { Tulsa, OK, USA }\end{array}$ & $\begin{array}{c}\text { Powder: zinc oxide }(75 \%), \\
\text { polymethacrylate }(20 \%) \\
\text { Liquid: eugenol }(99 \%), \text { acetic acid } \\
\text { (less than } 1 \%)\end{array}$ \\
\hline PRM & ProRoot MTA & $\begin{array}{l}\text { Dentsply Sirona, } \\
\text { Tulsa, OK, USA }\end{array}$ & $\begin{array}{l}\text { Powder: tricalcium silicate, } \\
\text { dicalcium silicate, bismuth oxide, } \\
\text { calcium sulfate, gypsum } \\
\text { Liquid: distilled water }\end{array}$ \\
\hline $\mathrm{BIO}$ & Biodentine & $\begin{array}{l}\text { Septodont, Saint } \\
\text { Maur des Faussés, } \\
\text { France }\end{array}$ & $\begin{array}{c}\text { Powder: tricalcium silicate, } \\
\text { dicalcium silicate, zirconium oxide, } \\
\text { calcium carbonate, calcium oxide, } \\
\text { dihydrate or gypsum } \\
\text { Liquid: distilled water, hydrosoluble } \\
\text { polymer, calcium chloride }\end{array}$ \\
\hline WRP & Well-Root PT & $\begin{array}{l}\text { Vericom, Chuncheon, } \\
\text { Korea }\end{array}$ & $\begin{array}{l}\text { Tricalcium silicate, dicalcium silicate, } \\
\text { zirconium oxide, polyethylene } \\
\text { glycol, polypropylene glycol, } \\
\text { calcium sulfate dihydrate }\end{array}$ \\
\hline
\end{tabular}

\subsection{Cell Viability}

The morphology of DPSCs was examined using a conventional optical microscope (LX-51, Olympus, Tokyo, Japan), and the viability of the DPSCs from each culture group was evaluated on days 1,3, and 7. For the 2D groups, the subculture of DPSCs that were on a 2D 24-well plate were cultured with different cement eluates. The cement eluate medium was refreshed every two days. The viability of the DPSCs was measured using the Cell Counting kit-8 (CCK-8, Dojindo, Tokyo, Japan) assay on days 1, 3, and 7. For this, $2.2 \mathrm{~mL}$ of CCK-8 solution was diluted at a 1:10 ratio with a basal medium and allowed to rest for $4 \mathrm{~h}$. Next, $10 \mu \mathrm{L}$ of the diluted solution was added to each well of a 2D 96-well plate. Each eluate was assessed in triplicate. The plate was wrapped in aluminum foil to prevent light exposure, and the absorbance was measured at $450 \mathrm{~nm}$ using a SpectraMax ELISA reader (Molecular Devices, Sunnyvale, CA, USA). The average absorbance of each set of three wells was considered for the statistical calculations. For the ULA groups, the subculture of DPSCs that were on a ULA 96-well plate were cultured with each cement eluate. The cement eluate medium was refreshed once every two days. The viability of the DPSCs was measured using the CellTiter-Glo (Promega, Madison, WI, USA) assay on days 1, 3, and 7. For this, $100 \mu \mathrm{L}$ of CellTiter-Glo reagent was added to each of the 96 wells. Each eluate was assessed in triplicate. The plate was shaken at a constant speed for $30 \mathrm{~min}$ to induce cell lysis. After this, the contents of the wells were transferred to a 96-well white plate and the luminescence was measured using a SpectraMax ELISA reader (Molecular Devices). The average absorbance of each set of wells was considered for the statistical calculations. 


\subsection{Alkaline Phosphate Activity Test}

To induce osteogenic differentiation, cement eluates were cultured with an osteogenic medium that contained $\alpha$-MEM with $10 \%$ FBS, gentamycin, dexamethasone $(10 \mathrm{nM})$, Lascorbic acid $(100 \mu \mathrm{M})$, and $\beta$-glycerophosphate $(10 \mathrm{mM})$. The negative control group was cultured in a basal medium, and the positive control group was cultured in an osteogenic medium. Cells were incubated for 1 week, with their respective media being replaced every 2-3 d. After 1 week, the cells were washed by diluting the medium. The alkaline phosphate (ALP) activity of DPSCs was measured using an alkaline phosphate assay kit (ab83369; Abcam, Cambridge, MA, USA). For this, $50 \mu \mathrm{L}$ of a p-nitrophenyl phosphate (p-NPP) solution was added to $80 \mu \mathrm{L}$ of each cement eluate. After $1 \mathrm{~h}$ at $25^{\circ} \mathrm{C}$ in the dark, $20 \mu \mathrm{L}$ of stop solution was added to each sample to stop the color reaction with p-NPP. The samples were then transferred to a new 96-well plate, and absorbance was measured at $405 \mathrm{~nm}$ using a SpectraMax ELISA reader (Molecular Devices), followed by its conversion into enzyme activity units. The protein value was then determined using the Bradford method. ALP activity was expressed as U/L protein.

\subsection{Alizarin Red S Staining}

DPSCs were seeded in a 24-well cell culture plate (SPL Life Science, Seongnam, Korea) at a density of $1.0 \times 10^{4}$ cells / well for the 2D group, and a Corning ${ }^{\circledR}$ Ultra-Low Attachment 96-well plate (Corning) at a density of $2.0 \times 10^{4}$ cells/well for the ULA group. DPSCs were cultured with an osteogenic medium containing $\alpha$-MEM with $10 \%$ FBS, gentamycin, dexamethasone $(10 \mathrm{nM})$, L-ascorbic acid $(100 \mu \mathrm{M})$, and $\beta$-glycerophosphate $(10 \mathrm{mM})$. The negative control group was cultured in a growth medium (control_GM), and the positive control group was cultured in an osteogenic medium (control_OM). Cells were incubated for 3 weeks, with their respective media being freshly replaced every 2-3 d. After 21 days, the medium was removed, and the cells were washed with PBS. DPSCs were fixed in 70\% ethanol and stained with $300 \mu \mathrm{L}$ of $2 \%$ alizarin red S staining reagent (LIFELINE Cell Tech, Frederick, MD, USA). After removing the staining reagent, a photograph was taken as it was before and qualitatively examined.

\subsection{Scanning Electronic Microscope (SEM)}

Samples of PRM, BIO, and WRP were shaped into four disks with a $2.5 \mathrm{~mm}$ thickness and with a $7 \mathrm{~mm}$ diameter using rubber molds. The DPSCs were directly seeded onto each disk at a density of $5 \times 10^{4}$ cells $/ \mathrm{mL}$. After $5 \mathrm{~d}$ of culturing, the disks with DPSCs were fixed in $3 \%$ glutaraldehyde for $4 \mathrm{~h}$, dehydrated in a graded series of ethanol up to $100 \%$, immersed in $100 \%$ hexamethyldisilazane, air dried, mounted on aluminum stubs, and sputter coated with gold/palladium (Polaron e5400 SEM Sputter Coating System; Bio-Rad, Kennett Square, PA, USA), before being visualized using SEM at magnifications of $\times 100$, $\times 400$, and $\times 1000$.

\subsection{Statistical Analysis}

Cell viability values were statistically analyzed using a two-way ANOVA and Tukey's honest significant difference (HSD) post hoc test. A two-way ANOVA was performed to determine the interaction between 'material type' and 'culturing time'. The results of the ALP activity test were statistically analyzed using a one-way ANOVA and Tukey's HSD post hoc test. The statistical significance was set at a confidence level of $95 \%$. Differences were considered statistically significant at $p<0.05$.

\section{Results}

\subsection{Morphology}

Figure 1 shows the morphology of 2D and ULA-cultured DPSCs with different cement eluates. The DPSCs exhibited fibroblastic morphology in the control, IRM, PRM, BIO, and WRP groups (Figure 1A-E). The DPSCs in the IRM group were not attached to the dish, but were floating in the medium (Figure 1B). The DPSCs that were cultured in 2D showed that 
the number of cells increased with time. Figure 1F-J shows the morphology of the DPSCs that were cultured in ULA plates with each cement eluate. The DPSCs in the control, PRM, $\mathrm{BIO}$, and WRP groups formed microspheres. These microspheres became smaller with increasing aggregation over time. In contrast, DPSCs in the IRM group had floating cells that did not form microspheres well (Figure 1G).

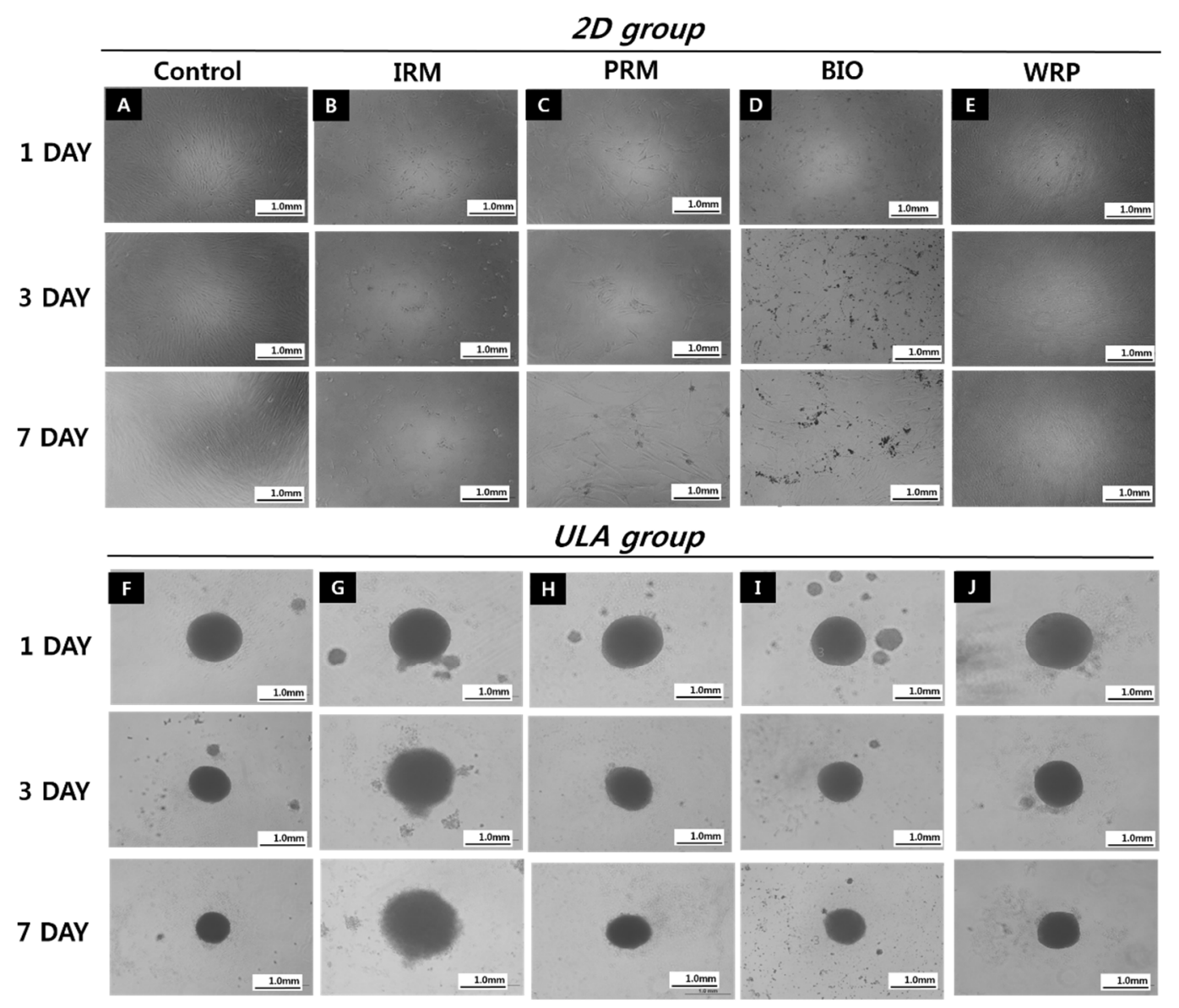

Figure 1. Morphology of DPSCs during seven days of incubation in 2D (A-E) and ULA (F-J) culture plates: (A,F) Control group; (B,G) IRM group; (C,H) PRM group; (D,I) BIO group; (E,J) WRP group.

\subsection{Cell Viability}

The results of the cell viability test of the 2D and ULA groups are presented in Tables 2 and 3. In both the 2D and ULA groups, PRM, BIO, and WRP exhibited significantly higher cell viability compared to IRM at all of the indicated time points, except for the ULA group on day $1(p<0.05)$. In the 2D group, cell viability in BIO was significantly lower than that in PRM and WRP on day $1(p<0.05)$. In the ULA group, BIO exhibited significantly higher cell viability than PRM and WRP throughout the study period $(p<0.05)$.

Table 2. Cell viability in $2 \mathrm{D}$ groups.

\begin{tabular}{cccc}
\hline \multicolumn{4}{c}{ (Percentage of Control, \%) } \\
\hline Group & 1 Day & 3 Day & 7 Day \\
\hline IRM & $2.56( \pm 0.51)^{\mathrm{A}}$ & $1.59( \pm 0.46)^{\mathrm{A}}$ & $5.78( \pm 0.16)^{\mathrm{A}}$ \\
PRM & $63.92( \pm 12.56)^{\mathrm{Ca}}$ & $82.48( \pm 6.07)^{\mathrm{Bb}}$ & $90.74( \pm 6.07)^{\mathrm{Cb}}$ \\
BIO & $25.79( \pm 8.39)^{\mathrm{Ba}}$ & $56.26( \pm 3.07)^{\mathrm{Bb}}$ & $79.90( \pm 2.11)^{\mathrm{Bb}}$ \\
WRP & $87.91( \pm 8.56)^{\mathrm{Db}}$ & $55.49( \pm 18.68)^{\mathrm{Ba}}$ & $87.68( \pm 4.21)^{\mathrm{BCb}}$
\end{tabular}

Values are written as means ( \pm standard deviation). Values with different capital and lowercase letters within each column and row represent statistically significant differences $(p<0.05)$. 
Table 3. Cell viability in ULA groups.

\begin{tabular}{cccc}
\hline \multicolumn{4}{c}{ (Percentage of Control, \%) } \\
\hline Group & 1 Day & 3 Day & 7 Day \\
\hline IRM & $106.09( \pm 12.01)^{\mathrm{A}}$ & - & - \\
PRM & $107.46( \pm 4.39)^{\mathrm{ABa}}$ & $130.04( \pm 9.06)^{\mathrm{Ab}}$ & $184.75( \pm 4.48)^{\mathrm{Bc}}$ \\
BIO & $135.59( \pm 17.75)^{\mathrm{Ba}}$ & $181.62( \pm 15.05)^{\mathrm{Bb}}$ & $228.81( \pm 6.78)^{\mathrm{Cc}}$ \\
WRP & $91.31( \pm 10.40)^{\mathrm{Aa}}$ & $144.09( \pm 13.34)^{\mathrm{Ab}}$ & $127.83( \pm 17.87)^{\mathrm{Ab}}$ \\
\hline
\end{tabular}

Values are written as means ( \pm standard deviation). Values with different capital and lowercase letters within each column and row represent statistically significant differences $(p<0.05)$.

\subsection{ALP Activity and Qualitative Alizarin Red S Staining Assay}

The results of the ALP activity test using the ab83369 ALP assay kit and statistical one-way ANOVA are shown in Table 4 . In both the 2D and ULA groups, the ALP activity of PRM was significantly higher than that of BIO and WRP $(p<0.05)$. Figure 2 presents the results of the alizarin red staining assay. Its application in the PRM and BIO groups resulted in intense staining, which was similar to the control OM group. WRP had some more mineralized nodules than the control GM. However, the generalized staining was much less than with other MTA groups. The ULA groups had stained microspheres, regardless of the experimental groups. However, the staining made it harder to examine the odontogenic and/or osteogenic differentiation, due to its morphological, three-dimensional, sphereforming characteristics.

Table 4. Alkaline phosphate activity in the experimental groups.

\begin{tabular}{ccc}
\hline & (U/L Protein) & \\
\hline Group & 2D & ULA \\
\hline Basal media & $1.68( \pm 0.02)^{\mathrm{A}}$ & $1.42( \pm 0.02)^{\mathrm{A}}$ \\
Osteogenic media & $3.21( \pm 0.78)^{\mathrm{C}}$ & $2.75( \pm 0.04)^{\mathrm{BC}}$ \\
PRM & $3.01( \pm 2.78)^{\mathrm{C}}$ & $3.25( \pm 0.15)^{\mathrm{C}}$ \\
BIO & $2.03( \pm 0.14)^{\mathrm{AB}}$ & $2.63( \pm 0.44)^{\mathrm{B}}$ \\
WRP & $2.69( \pm 0.52)^{\mathrm{BC}}$ & $2.54( \pm 0.01)^{\mathrm{B}}$ \\
\hline
\end{tabular}

Values are written as means ( \pm standard deviation). Values with different capital and lowercase letters within each column and row represent statistically significant differences $(p<0.05)$.

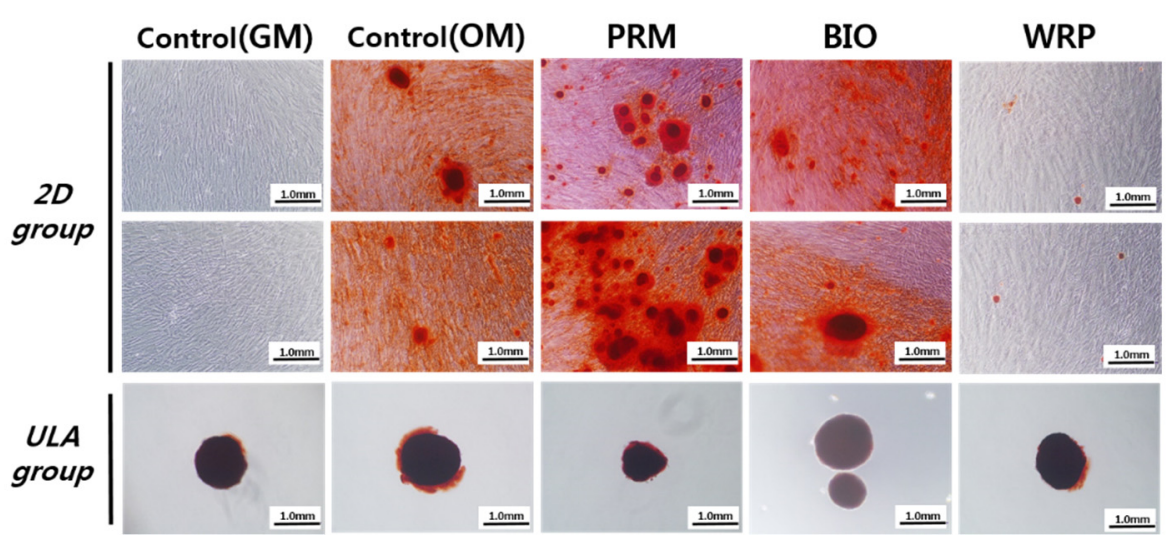

Figure 2. Alizarin red S staining of the DPSCs that were cultured in 2D and ULA culture plates for 21 days.

\subsection{Scanning Electron Microscopy}

Figure 3 shows the scanning electron microscopy (SEM) images of the morphology of the DPSCs on the surfaces of the different materials after $120 \mathrm{~h}$ of culture. These images revealed that the DPSCs were able to attach to each material. They covered the surface of PRM, BIO, and WRP with spindle-shaped, flattened fibroblastic morphologies 
(red asterisks in Figure 3). However, in contrast to PRM and BIO, on the WRP surface, fibroblastic morphology was barely visible, and cloud-like clustering of dead DPSCs was observed extensively under low magnification (red arrow in Figure 3). The DPSCs on PRM appeared flatter and broader in shape than the other groups.

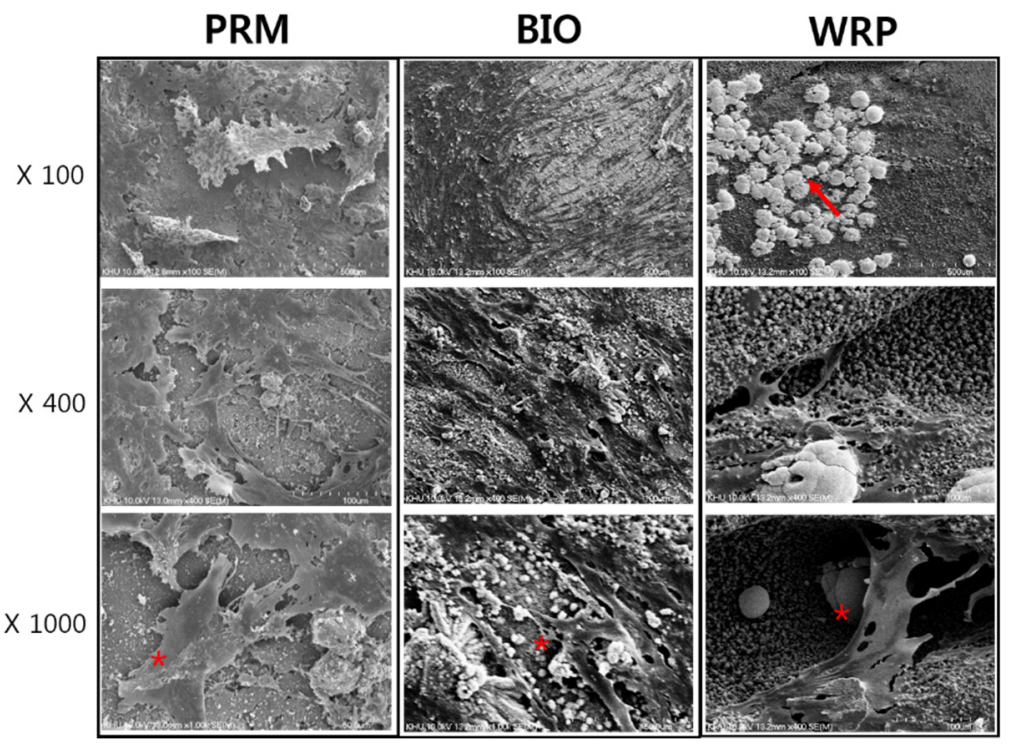

Figure 3. Scanning electron microscopic images of the DPSCs that were on the surface of different MTA materials.

\section{Discussion}

In regenerative endodontics, biocompatible materials come into direct contact with blood clots, which act as natural scaffolds that contain DPSCs, and they regenerate dentinpulp complexes through an appropriate biological response. Thus, the requisites for biocompatible materials in regenerative endodontics are that they be non-toxic to dental pulp tissues and capable of inducing dentin repair or regeneration. MTAs are commonly used as biocompatible materials. In this study, DPSCs were exposed to the eluates of MTA cements to simulate the conditions of regenerative endodontics, and cell viability was measured using the CCK-8 assay for the 2D group and the CellTiter-Glo assay for the ULA group with observations of the morphology of the attached DPSCs or DPSC microspheres. Almost all of the groups of cells exhibited normal morphology and structure (Figure 1). In the 2D culture, the DPSCs exhibited a fibroblastic morphology, as has been observed in a previous study [12]. In contrast, when DPSCs were cultured in ULA, one large spheroid was observed. The diameter of this spheroid of DPSCs showed a tendency to decrease because the cells clustered together over time. In the IRM group, DPSCs were not attached, but remained suspended when cultured in 2D; when cultured in ULA, they did not aggregate with each other and instead spread apart. This is consistent with the low cell viability observed in the treatment with the IRM eluate.

The CCK-8 assay measures the reduction of tetrazolium, and the change from WST- 8 to formazan, which occurs through enzyme activity in viable cells and has a specific color [17]. However, in the ULA group, where cells are organized as microspheres, it is difficult to accurately measure the change in color when conducting tetrazolium reduction analysis. This is because the tight cell-cell interactions in compact spheroids hamper the subsequent reduction to formazan, leading to an under-representation of the actual number of healthy cells [18]. CellTiter-Glo is a luminescence assay that determines the number of viable cells in culture by quantifying the total ATP content. In the results of our study, cells treated with IRM eluate exhibited significantly lower absorbance in CCK-8 and CellTiter-Glo assays; the CCK-8 assay showed moderate absorbance values in the presence of all other MTA test groups. This difference was probably a result of the differences in the composition of 
the materials. A previous study reported that zinc oxide eugenol, a major component of IRM, has lower biocompatibility than calcium silicate, a major component of MTAs [19]. We performed the cell viability test, taking measurements on days 1, 3 and 7. According to the previous investigation of ours [12] and the preliminary pilot study, day 3 and day 5 showed a similar level of cell viability regardless of the experimental groups. Thus, we decided to investigate the immediate, early (day 1 and 3), and mature (day 7) stages of cell viability without providing the day 5 information.

In this study, the odontoblastic differentiation and mineralization effect of the experimental groups were investigated using ALP activity test and qualitative alizarin red staining assay. ALP activity is a biochemical marker for osteoblasts and the formation of new bone. In regenerative endodontic procedures, DPSCs differentiate into osteoblasts and form mineralized tissues. ALP releases free phosphate ions, which react with calcium ions to form a calcium phosphate precipitate, the molecular unit of hydroxyapatite. A previous study reported that ALP exhibited a pronounced expression one week after its induction, and it can be considered to be an early marker of osteo/odontogenic differentiation [20]. In this study, IRM was excluded because it had little cell viability, and basal media and osteogenic media were set as negative and positive control groups, respectively. There were no differences between the 2D and ULA groups, and the ALP activity in the cells that were grown with all types of MTA was comparable to that of the control group. In particular, PRM stimulated the highest levels of ALP in DPSCs, which was consistent with a previous report that found that PRM exhibited a high level of ALP activity [21].

We utilized qualitative alizarin red staining to evaluate the mineralization of the extracellular matrix for the 2D- and ULA-cultured DPSCs (Figure 2). For the 2D groups, the PRM and BIO groups showed a greater increase in staining compared to control group, which differed to the WRP group. PRM and BIO are currently referred to as hydraulic calcium silicate cements (CSCs), and a recently released systematic review reported that the application of these MTA-type CSCs led to no major differences in biocompatibility and odontogenic effect when applied to human pulp-derived cells $[22,23]$. These reports were coincident with our study regardless of the culture condition, though our study examined the effect of various CSCs on the two- and three-dimensionally cultured DPSCs. To the best of our knowledge, the three-dimensionally cultured microsphere-forming DPSCs have not been investigated very much, so our findings might provide the potential evidence that supports the utilization of the differently cultured DPSCs in endodontic regenerative research or their future application in clinical procedures.

Generally, the alizarin red staining assay is used to quantitatively analyze the amount of odontogenic differentiation. However, due to the different characteristics of the threedimensionally cultured microspheres of DPSCs, the quantitative comparison between 2D and 3D culture methods could not be conducted. To negotiate the effect of the culture methods and the different type of the MTA materials, RNA- or DNA-level laboratory examination, such as RNA sequencing, is required due to the methodological differences in the process of analysing the three-dimensionally cultured cells.

Although our study did not analyze the degree of the quantitative mineralization staining, WRP exhibited much less mineralization staining after undergoing 21 days in the odontogenic differentiation-conditioned medium compared to PRM and BIO. As mentioned previously, WRP is the newly formulated premixed CSC, which is a ready-to-use bioceramic paste. According to the manufacturer's information, the materials are composed of two major groups of chemical components. The first are tricalcium silicate, dicalcium silicate, and zirconium oxide, which are most similar to that of other traditional MTA-type CSCs; and the second are polyethylene glycol, polypropylene glycol, and calcium sulfate dehydrate, which are different from conventional materials. Although our study has not explored the physicochemical and compositional properties of the experimental materials, the premixed-type CSCs might not have the chemical composition necessary to induce the mineralization or odontogenic differentiation. 
The biomaterials that are used in endodontics make direct contact with pulp and periodontal tissues. Cell attachment to a material surface is a good indication that the material is biocompatible. Furthermore, if the material stimulates cell proliferation or mineralization, then it is likely to promote the healing process. Observing the material surface with SEM (Figure 3) can confirm whether the DPSCs adhere to it. In the PRM and BIO groups, DPSCs adhered to the surface well, whereas in the WRP group, only a cloud of dead cells was observed, and almost no cells with fibroblastic morphology were observed. Further examinations, such as RNA extraction and PCR assay and Western blot analysis with the specific markers (DSPP, DMP-1, Runx2, FGF-1 and VEGF), scratch wound healing assay, and RNA sequencing, might provide more detailed scientific evidence, which could contribute to the utilization of the DPSC microspheres in laboratory and clinical settings.

Within the limitations of this study, our results indicate that the DPSCs that were cultured in ULA showed a level of viability and mineralization capacity similar to those cultured in 2D after reacting with some MTAs, including PRM, BIO, and WRP. This suggests that the biocompatibility of MTAs with 3D-cultured DPSCs is comparable to that of 2Dcultured DPSCs. Future investigations using supplemental 3D culture methods, such as hanging drop, magnetic levitation, and gel embedding, are required to understand the precise interaction between MTAs and DPSCs in a regenerative endodontics environment.

\section{Conclusions}

None of the MTA groups affected the microsphere-forming characteristics of the DPSCs that were cultured in ULA. The DPSCs that had been cultured in ULA exhibited high cell viability in all of the MTA groups. The mineralization potential was found to be good in all MTA groups, showing both significant osteogenic differentiation and high ALP activity of the DPSCs that had been cultured in ULA using an osteogenic medium. In particular, the PRM group showed significantly higher ALP activity than the other MTA groups.

Author Contributions: J.-H.J. designed, planned, and coordinated the study; S.-H.K. and J.-H.J. wrote the manuscript; S.-H.K. and H.-J.J. participated in the experiments; B.-N.L. and H.-S.L. played a substantial role in the experimental design and material selection; H.-J.K., S.-Y.K. and D.-S.K. contributed to the interpretation of the results and the discussion. All authors have read and agreed to the published version of the manuscript.

Funding: This research was supported by the Bio \& Medical Technology Development Program of the National Research Foundation (NRF) and funded by the Korean government (MSIP \& MOHW) NRF (No.2019R1G1A1100082).

Institutional Review Board Statement: Not applicable.

Informed Consent Statement: Not applicable.

Data Availability Statement: The data presented in this study are available on request from the corresponding author.

Conflicts of Interest: The authors declare no conflict of interest.

\section{References}

1. Gomes-Cornélio, A.L.; Rodrigues, E.M.; Salles, L.P.; Mestieri, L.B.; Faria, G.; Guerreiro-Tanomaru, J.M.; Tanomaru-Filho, M. Bioactivity of MTA Plus, Biodentine and an experimental calcium silicate-based cement on human osteoblast-like cells. Int. Endod. J. 2017, 50, 39-47. [CrossRef]

2. Torabinejad, M.; Hong, C.U.; Lee, S.J.; Monsef, M.; Pitt Ford, T.R. Investigation of mineral trioxide aggregate for root-end filling in dogs. J. Endod. 1995, 21, 603-608. [CrossRef]

3. Lee, S.J.; Monsef, M.; Torabinejad, M. Sealing ability of a mineral trioxide aggregate for repair of lateral root perforations. J. Endod. 1993, 19, 541-544. [CrossRef]

4. Chen, I.; Salhab, I.; Setzer, F.C.; Kim, S.; Nah, H.D. A New Calcium Silicate-based Bioceramic Material Promotes Human Osteoand Odontogenic Stem Cell Proliferation and Survival via the Extracellular Signal-regulated Kinase Signaling Pathway. J. Endod. 2016, 42, 480-486. [CrossRef] [PubMed]

5. Torabinejad, M.; Chivian, N. Clinical applications of mineral trioxide aggregate. J. Endod. 1999, 25, 197-205. [CrossRef] 
6. Parirokh, M.; Torabinejad, M. Mineral trioxide aggregate: A comprehensive literature review—Part I: Chemical, physical, and antibacterial properties. J. Endod. 2010, 36, 16-27. [CrossRef]

7. Song, J.S.; Mante, F.K.; Romanow, W.J.; Kim, S. Chemical analysis of powder and set forms of Portland cement, gray ProRoot MTA, white ProRoot MTA, and gray MTA-Angelus. Oral Surg. Oral Med. Oral Pathol. Oral Radiol. Endodontol. 2006, 102, 809-815. [CrossRef]

8. About, I. Biodentine: From biochemical and bioactive properties to clinical applications. G. Ital. Di Endod. 2016, 30, 81-88. [CrossRef]

9. Murray, P.E.; Garcia-Godoy, F.; Hargreaves, K.M. Regenerative endodontics: A review of current status and a call for action. J. Endod. 2007, 33, 377-390. [CrossRef] [PubMed]

10. O'Brien, F.J. Biomaterials \& scaffolds for tissue engineering. Mater. Today 2011, 14, 88-95.

11. Malhotra, N.; Mala, K. Regenerative endodontics as a tissue engineering approach: Past, current and future. Aust. Endod. J. 2012, 38, 137-148. [CrossRef] [PubMed]

12. Bu, N.U.; Lee, H.S.; Lee, B.N.; Hwang, Y.C.; Kim, S.Y.; Chang, S.W.; Choi, K.K.; Kim, D.S.; Jang, J.H. In Vitro Characterization of Dental Pulp Stem Cells Cultured in Two Microsphere-Forming Culture Plates. J. Clin. Med. 2020, 9, 242. [CrossRef]

13. Volponi, A.A.; Pang, Y.; Sharpe, P.T. Stem cell-based biological tooth repair and regeneration. Trends Cell Biol. 2010, 20, 715-722. [CrossRef]

14. Baker, B.M.; Chen, C.S. Deconstructing the third dimension: How 3D culture microenvironments alter cellular cues. J. Cell Sci. 2012, 125 Pt 13, 3015-3024. [CrossRef] [PubMed]

15. Kulan, P.; Karabiyik, O.; Kose, G.T.; Kargul, B. Biocompatibility of Accelerated Mineral Trioxide Aggregate on Stem Cells Derived from Human Dental Pulp. J. Endod. 2016, 42, 276-279. [CrossRef] [PubMed]

16. Lee, S.H.; Inaba, A.; Mohindroo, N.; Ganesh, D.; Martin, C.E.; Chugal, N.; Kim, R.H.; Kang, M.K.; Park, N.H.; Shin, K.H. Three-dimensional Sphere-forming Cells Are Unique Multipotent Cell Population in Dental Pulp Cells. J. Endod. 2017, 43, 1302-1308. [CrossRef] [PubMed]

17. Riss, T.L.; Moravec, R.A.; Niles, A.L.; Duellman, S.; Benink, H.A.; Worzella, T.J.; Minor, L. Cell Viability Assays. In Assay Guidance Manual; Markossian, S., Grossman, A., Brimacombe, K., Arkin, M., Auld, D., Austin, C.P., Baell, J., Chung, T.D.Y., Coussens, N.P., Dahlin, J.L., et al., Eds.; Eli Lilly \& Company and the National Center for Advancing Translational Sciences: Bethesda, MD, USA, 2004.

18. Walzl, A.; Unger, C.; Kramer, N.; Unterleuthner, D.; Scherzer, M.; Hengstschlager, M.; Schwanzer-Pfeiffer, D.; Dolznig, H. The Resazurin Reduction Assay Can Distinguish Cytotoxic from Cytostatic Compounds in Spheroid Screening Assays. J. Biomol. Screen 2014, 19, 1047-1059. [CrossRef]

19. Ma, J.; Shen, Y.; Stojicic, S.; Haapasalo, M. Biocompatibility of two novel root repair materials. J. Endod. 2011, 37, 793-798. [CrossRef]

20. Bakopoulou, A.; Leyhausen, G.; Volk, J.; Tsiftsoglou, A.; Garefis, P.; Koidis, P.; Geurtsen, W. Comparative analysis of in vitro osteo/odontogenic differentiation potential of human dental pulp stem cells (DPSCs) and stem cells from the apical papilla (SCAP). Arch. Oral. Biol. 2011, 56, 709-721. [CrossRef]

21. Chang, S.W.; Lee, S.Y.; Kum, K.Y.; Kim, E.C. Effects of ProRoot MTA, Bioaggregate, and Micromega MTA on odontoblastic differentiation in human dental pulp cells. J. Endod. 2014, 40, 113-118. [CrossRef] [PubMed]

22. Niu, L.-N.; Jiao, K.; Zhang, W.; Camilleri, J.; Bergeron, B.E.; Feng, H.L.; Mao, J.; Chen, J.H.; Pashley, D.H.; Tay, F.R. A review of the bioactivity of hydraulic calcium silicate cements. J. Dent. 2014, 42, 517-533. [CrossRef] [PubMed]

23. Emara, R.; Elhennawy, K.; Schwendicke, F. Effects of calcium silicatae cements on dental pulp cells: A systematic review. J. Dent. 2018, 77, 18-36. [CrossRef] [PubMed] 